\title{
Fluid management in diabetic ketoacidosis
}

\section{D Inward, T L Chambers}

\section{Have we got it right yet?}

Y oung people with insulin dependent diabetes mellitus are three times more likely to die in childhood than the general population. ${ }^{1}$ Despite advances in management over the past 20 years, the incidence of mortality associated with diabetic ketoacidosis (DKA) remains unchanged. Cerebral oedema is the predominant cause of this mortality; young children are particularly at risk, with an incidence of $0.7-1 \%$ of episodes of DKA. ${ }^{23}$ The mortality appears to be greatest among patients at first presentation, ${ }^{134}$ if there has been a long history of symptoms prior to admission, ${ }^{3}$ and during the first 24 hours of treatment. ${ }^{4}$ In a recently published retrospective multicentre analysis of children with DKA, low $\mathrm{pCO}_{2}$ levels and high serum sodium concentration at presentation were identified as particular risk factors for the development of cerebral oedema, together with bicarbonate therapy. ${ }^{5}$ However, in the accompanying editorial, Dunger and Edge point out that this may simply be revealing an association between severe DKA and dehydration and the risk of cerebral oedema. ${ }^{6}$ The pathogenesis of cerebral oedema remains poorly understood but there may be many contributing factors. ${ }^{7}$

The aim of management of DKA is to restore metabolic homoeostasis while minimising the risks of complications including hypoglycaemia, hypokalaemia, cardiac failure, and in children the development of cerebral oedema. How best to achieve this remains contentious, with particular controversy centred on optimal fluid management. The most appropriate volume, type, and rate of fluid to be given have all been the subject of debate. A survey in 1994 of UK paediatricians found a threefold variation in the amount of fluid recommended within the first 12 hours. ${ }^{8}$ Since then national guidelines have been developed by the British Society for Paediatric Endocrinology (BSPE). However, it remains uncertain whether the recommended strategy, using a volume calculated by "maintenance plus deficit", is the safest and most appropriate way to manage rehydration in children with DKA. We suggest that childhood DKA may be considered and treated as a variant of renal electrolyte disorder with hypertonic dehydration.

\section{FLUID AND ELECTROLYTE LOSSES}

The fluid and electrolyte losses of DKA are predominantly caused by hyperglycaemia with resultant glycosuria and osmotic diuresis. In addition, the kidney has a low threshold for ketoacids, which are excreted into the urine with an accompanying cation, further exacerbating the electrolyte loss. Relative acute dehydration with a larger sodium loss will result primarily in intravascular dehydration, but a more prolonged development will result in a greater degree of intracellular dehydration, with relative maintenance of intravascular volume, particularly if the patient remains polydipsic. ${ }^{9}$ There are thus wide variations in fluid, acid-base, and electrolyte deficiencies, during both development and treatment of DKA, yet few protocols acknowledge this.

There is clear evidence that volume depletion triggers the release of counter regulatory hormones including catecholamines, growth hormone, and cortisol as well as renin, aldosterone, and arginine vasopressin. ${ }^{10}{ }^{11}$ These hormones, whose combined actions are directed towards preserving intravascular volume, also cause insulin resistance. An important effect of fluid administration is therefore reducing the stimulus for the secretion of the counter regulatory hormones. Indeed it has been shown that plasma glucose starts to fall following fluid administration prior to giving insulin, although insulin is required for correction of the metabolic acidosis. ${ }^{12}$

\section{RISK FACTORS FOR CEREBRAL OEDEMA}

The importance of prompt restoration of the circulating volume for patients presenting with DKA is clear. However, the observation that symptomatic cerebral oedema often occurs several hours after treatment has begun, usually at a time when plasma glucose has started to fall and metabolic acidosis to improve, has led to concern that the treatment itself may contribute to the development of cerebral oedema. There have been few case controlled studies of cerebral oedema complicating DKA and there is conflicting evidence as to the role of iatrogenic factors in fatal cases. ${ }^{6}{ }^{13} \mathrm{How}-$ ever, excessive rates of fluid administration, particularly early in resuscitaion, ${ }^{14}$ and failure of plasma sodium to rise as plasma glucose declines, have been identified as risk factors.

Studies using computed tomography suggest that subclinical cerebral swelling may be a common occurrence in children presenting with DKA, ${ }^{15}$ although this has been disputed. ${ }^{17}$ If cerebral swelling is a relatively common event it is not clear why only a minority progress to herniation. In addition, fatal herniation has occurred in the absence of any fluid therapy. Therefore, although clearly not the only cause, it seems reasonable to conclude that fluid management may be a factor contributing to the development of overt cerebral oedema. On the basis of the available evidence it would seem prudent to avoid rapid rates of fluid infusion and to maintain serum sodium concentrations in order to avoid the potential danger of excess free water administration which may exacerbate the development of symptomatic brain swelling.

Failure to follow guidelines for the management of DKA has been well documented. ${ }^{18}$ Of particular concern is the evidence that clinicians have been consistently shown to overestimate the degree of dehydration in this condition. ${ }^{19}{ }^{20}$ Nevertheless, the assumption underlying most DKA protocols is that rehydration is desirable and achievable in 24 hours, using a volume calculated by "maintenance plus deficit".

\section{THE NEED FOR CHANGE?}

From a renal perspective the term "maintenance fluid" is a misnomer. True maintenance fluid volume in children is the sum of the insensible losses plus the obligate urine production and is probably only $40 \%$ of recommended amounts. ${ }^{21}$ Water requirements are related to energy expenditure. The recommended "maintenance" volumes are based on calculations of insensible losses (estimated as $50 \mathrm{ml} / 100 \mathrm{cal} /$ day) plus $66.7 \mathrm{ml} / 100 \mathrm{cal} /$ day to replace urine output. ${ }^{22}$ This allows for production of isotonic urine. As normally functioning kidneys are capable of fourfold concentraton of the glomerular filtrate, "maintenance fluids" will provide sufficient volume for both insensible and obligate losses as well as the extra needed for rehydration. A 5\% dehydrated child with normally functioning kidneys can be rehydrated using "maintenance" fluid only_albeit slowly.

In non-diabetic dehydration it is customary to assess whether the dehydration is hypertonic, hypotonic, or isotonic 
and whether there is simple contraction of the extracellular compartment or whether compensatory mechanisms are operating.

It is accepted that safe therapy of hypertonic dehydration should be both prolonged and cautious to avoid the development of fits and cerebral oedema during rehydration. ${ }^{23} 24$

The cells of the central nervous system protect cell volume under hyperosmolar conditions by producing intracellelular osmotically active molecules, "idiogenic osmoles". ${ }^{25}$ Once formed these molecules dissipate slowly. Rapid reduction of plasma osmolality by the administration of free water under these conditions will create a gradient, causing movement of water into brain cells and thus brain swelling. These considerations probably apply equally to hypernatraemic dehydration and diabetic ketoacidosis.

We suggest that the treatment of dehydration of severe DKA should be based on the general principles used to treat dehydration combined with renal failure, taking into account the additional relatively brief ${ }^{19}$ problem of an osmotic diuresis. Halting the decompensation process is the initial therapeutic goal, followed by provision of sufficient fluid to allow the establishment of renal and hormonal homoeostasis over a period of time comparable to that taken for the problem to develop. There is no evidence that the use of modest fluid regimens is harmful. ${ }^{26}$ Harris and colleagues $^{1928}$ have shown that in 149 episodes of childhood DKA, rehydration over 48 hours was associated with "no deaths or near death episodes", and Johnston reported that in adults a regimen of $48-72 \mathrm{ml} / \mathrm{kg} /$ day was safe. ${ }^{29}$

\section{A PROPOSAL}

Twenty or so years ago there was a revolution in the management of DKA with the introduction of continuous intravenous insulin infusion. Perhaps it is now time for another review, not only of the fluid management, but also of all other aspects. There are several interested parties-endocrinologists, nephrologists, general paediatricians, and specialists in emergency medicine. We wonder whether a summit meeting should be convened to consider this; perhaps to commission research (the NHS and the corporate spirit of British paediatrics are ideal for such multicentre studies) and propose a second revolution in DKA management. Who will step forward?

\section{ACKNOWLEDGEMENTS}

We would like to thank Dr Fiona Cowan, Dr Mary McGraw, and Mrs Rachel Owen for their invaluable help in the preparation of the manuscript.

Arch Dis Child 2002;86:443-445

\section{Authors' affiliations}

C D Inward, T L Chambers, Bristol Royal Hospital for Children, Bristol, UK

Correspondence to: $\operatorname{Dr} C$ D Inward, Bristol Royal Hospital for Children, Upper Maudlin Street, Bristol BS2 8BJ, UK

\section{REFERENCES}

1 Edge JA, Ford-Adams ME, Dunger DB. Causes of death in children with insulin dependent diabetes 1990-96. Arch Dis Child 1999;81:318-23.

2 Edge JA, Hawkins MM, Winter DL, et al. Incidence presentation management and outcome of cerebral oedema associated with diabetic ketoacidosis (DKA) in Great Britain. Arch Dis Child 1999;80(suppl 1):A1 1

3 Bello FA, Sotos JF. Cerebral oedema in diabetic ketoacidosis in children. Lancet 1990;336:64

4 Rosenbloom AL. Intracerebral crises during treatment of diabetic ketoacidosis. Diabetes Care 1990;13:22-33

5 Glaser N, Barnett P, McCaslin I, et al. Risk factors for cerebral edema in children with diabetic ketoacidosis. N Engl J Med 2001;344:264-9

6 Dunger DB, Edge JA. Predicting cerebral oedema during diabetic ketoacidosis. N Engl J Med 2001;344:302-3

7 Edge JA. Cerebral oedema during treatment of diabetic ketoacidosis: are we any nearer finding a cause? Diabetes Metab Res Rev 2000;16:316-24

8 Edge JA, Dunger DB. Variations in the management of diabetic ketoacidosis in children. Diabet Med 1994;11:984-6.

9 Adrogue $\mathrm{HJ}$, Wilson $\mathrm{H}$, Boyd $\mathrm{AE}$, et al. Plasma acid-base patterns in diabetic ketoacidosis. N Engl J Med 1982;307:1603-10.

10 Tulassay T, Rascher W, Korner A, et al. Atrial natriuretic peptide and other vasoactive hormones during treatment of severe diabetic ketoacidosis in children. J Pediatr 1987; 111:329-34.

11 Luzi L, Barrett EJ, Groop LC, et al. Metabolic effects of low-dose insulin therapy on glucose metabolism in diabetic ketoacidosis. Diabetes 1988;37:1470-7

12 Owen OE, Licht JH, Sapir DG. Renal function and effects of partial rehydration during diabetic ketoacidosis. Diabetes 1981;30:510-18.

13 Mahoney CP, Vleck BW, DelAguila M. Risk factors for developing brain herniation during diabetic ketoacidosis. Pediatr Neurol 1999;21:721-7.

14 Rosenbloom AL, Schatz DA, Krischer JP, et al. Therapeutic controversy: prevention and treatment of diabetes in children. J Clin Endocrinol Metab 2000;85:494-522.

15 Krane EJ, Rockoff MA, Wallman JK, et al. Subclinical brain swelling in children during treatment of diabetic ketoacidosis. N Engl J Med 1985;312:1147-51.

16 Hoffman WH, Steinhart CM, ElGammal T, et al. Cranial CT in children and adolescents with diabetic ketoacidosis. Am J Neuroradiol 1988;9:733-9

17 Smedman L, Escobar R, Hesser U, et al. Subclinical cerebral oedema does not occur regularly during treatment for diabetic ketoacidosis. Acta Pediatr 1997;86: 1 172-6.

18 Singh RK, Perros P, Frier BM. Hospital management of diabetic ketoacidosis: are clinical guidelines implemented effectively? Diabet Med 1997;14:482-6.

19 Harris GD, Fiordalisi I, Harris WL, et al. Minimizing the risk of brain herniation during treatment of diabetic ketoacidemia: a retrospective and prospective study. J Pediatr 1990; 117:22-31.

20 Grove LM, Nobel-Jamieson CM, Barnes ND et al. Assessment of dehydration, fluid balance and insulin requirements in diabetic ketoacidosis. Proc Br Paediatr Assoc Annu Meet 1995;67:26.
21 Molteni KH. Initial management of hypernatraemic dehydration in the breastfed infant. Clin Pediatr 1994;33:731-40.

22 Holliday MA, Segar WE. The maintenance need for water in parenteral fluid therapy. Pediatrics 1957; 19:823-32.

23 Hollidat $M$. The evolution of therapy for dehydration: should deficit therapy still be taught? Pediatrics 1996;98:171-7.

24 Oddie S, Richmond S, Coultard M. Hypernatraemic dehydration and breast feeding: a population study. Arch Dis Child 2001;85:318-20.

25 Lee JH, Arcinue E, Ross BD. Organic osmolytes in the brain of an infant with hypernatremia. N Engl J Med 1994;331:439-42.

26 Lebovitz HA. Diabetic ketoacidosis. Lancet 1995;345:767-71

27 Adrogue H, Barrero J, Eknoyan G. Salutary effects of modest fluid replacement in the treatment of adults with ketoacidosis. JAMA 1989;262:2108-13.

28 Harris GD, Fiordalisi I. Physiologic management of diabetic ketoacidemia. A 5 -year prospective pediatric experience in 231 episodes. Arch Pediatr Adolesc Med 1994;148: 1046-52.

29 Johnston C. Fluid replacement in diabetic ketoacidosis. BM 1992;305:522.

COMMENTARY

$\mathrm{T}$ he treatment of the child with diabetic ketoacidosis (DKA) is unfortunately not based on very much evidence, but rather on anecdote and precedent. Fluid management has developed over several decades, with changes occurring gradually in an attempt to avoid complications (initially hypokalaemia, more recently cerebral oedema), rather than a logical approach to the particular fluid and electrolyte deficits. This has resulted in some anomalies in the management of DKA, and Inward and Chambers have now opened the debate about whether a revolution in fluid and electrolyte management is necessary.

DKA is associated with severe fluid losses. Much of the information about the specific fluid and electrolyte deficits in DKA was obtained in the 1930s from work on two young men with diabetes whose fluid and electrolyte balance was studied in great detail before, while, and after they were allowed experimentally to become ill with DKA. ${ }^{1}$ The reported fluid deficit appeared to be approximately half from the intracellular and half from the extracellular compartments. ${ }^{1-3}$ This work formed the basis for most of the DKA management protocols in existence. ${ }^{34}$ For obvious reasons, these studies have never been repeated in children, and yet body composition and fluid requirements are very different from those in adults.

Moreover, as Inward and Chambers point out, factors such as the duration of the episode of DKA will produce wide variations in fluid and electrolyte deficiencies. The water deficit is made up of varying combinations from the osmotic 
diuresis, vomiting, and hyperventilation. Sodium losses are also variable, depending on the predominating fluid type lost and on the adequacy of renal perfusion. There will also usually have been increased oral consumption of fluids of different compositions, which may affect the presenting fluid and electrolyte picture. ${ }^{5}$ It may not therefore be appropriate to treat all children with identical fluid regimens, particularly protocols extrapolated largely from studies in adults.

In particular, it is very difficult to quantify sodium losses. Plasma sodium levels may be low, normal, or high, depending more on renal perfusion and duration of polyuria than on total body losses. However, in current guidelines, the amount of sodium given as normal saline early during rehydration (after the resuscitation period) depends on the length of time it takes for the blood glucose to reach $12 \mathrm{mmol} / \mathrm{l}$, taking no account of sodium requirement. This may result either in giving too little sodium relative to water if the blood glucose falls too fast, or conversely giving too much if the blood glucose falls too slowly. This aspect certainly does require review.

Over the years, adult oriented protocols have been adapted to reduce the risk of various complications. For example, hypoglycaemia has largely been avoided by the use of low dose insulin infusions, ${ }^{67}$ and hypokalaemia by the use of early and adequate potassium replacement. ${ }^{8}$ A similar process has been occurring in an attempt to avoid cerebral oedema, the main cause of morbidity and mortality. The cause of this devastating complication is not known, but the timing of most episodes has suggested that it may be related to the fluid management. Furthermore, several studies (although not randomised controlled trials, or even casecontrol studies) have suggested that factors in the fluid and electrolyte management might play a part in the aetiology. ${ }^{9-11}$ This has led to changes in the protocols, such as slowing down of fluid replacement, lower volumes of "maintenance" fluids, avoidance of sodium bicarbonate administration, and close observation of plasma sodium concentration. All of these adaptations should theoretically make the protocols "safer", yet there is no evidence that the incidence of cerebral oedema has changed over the past 20 years. $^{12}$

There is furthermore no evidence that adopting the type of rehydration and electrolyte replacement scheme suggested by Inward and Chambers will have any impact on the incidence of cerebral oedema. In the quoted study of Harris and Fiordalisi in which children were rehydrated slowly with sufficient sodium to produce a gradual rise in plasma sodium concentrations, although there were "no deaths or near death episodes", there were still six children in whom behaviour change or increasing obtundation were treated with mannitol. ${ }^{11}$ It may therefore be increased vigilance and early rescue therapy which prevented sequelae from cerebral oedema rather than the type of fluid and electrolyte therapy. ${ }^{13}$ Inward and Chambers give very little detail of how a "renal" rehydration schedule would differ from current DKA guidelines which frequently do suggest a more protracted rehydration period.

Any guidelines for fluid and electrolyte management of DKA in children must be simple to calculate. Although no one would disagree with Nabarro et al that "the treatment of severe diabetic ketoacidosis should not be left to the house physician", ${ }^{2}$ it is generally the tired junior doctor who works out the fluid prescription. Given the plethora of DKA protocols previously in existence, and the complex nature of many of their calculation methods, ${ }^{14}$ it would not be in the interests of patients or doctors to produce guidelines which require very complicated calculations. We have only recently started in this country to adopt National (BSPED recommended) Guidelines, and have yet to see whether this will impact on the incidence of cerebral oedema.

Thus, although there are reservations, Inward and Chambers are right to open this debate. There are other anomalies in the treatment of DKA to be addressed by the proposed multidisciplinary discussion group, for which I would be delighted to step forward. However, until we have further research, which must be national or even international and collaborative, discussion (even involving many disciplines) will not provide us with all the answers.

John Raddliffe Hospital, Headley Way, Headington, Oxon OX3 9DU, UK

Arch Dis Child 2002;86:443-445

\section{REFERENCES}

1 Atchley DW, Loeb RF, Richards DW, et al. On diabetic acidosis. A detailed study of electrolyte balances following the withdrawal and reestablishment of insulin therapy. J Clin Invest 1933;12:297-326.

2 Nabarro JDN, Spencer AG, Stowers JM. Treatment of diabetic ketoacidosis. Lancet 1952;i:983-9.

3 Hillman K. Fluid resuscitation in diabetic emergencies - a reappraisal. Intensive Care Med 1987;13:4-8

4 WaldhausI W, Kleinberger G, Korn A, et al. Severe hyperglycaemia: effects of rehydration on endocrine derangements and blood glucose concentration. Diabetes 1979;28:577-84.

5 Vanelli M, Chairi G, Ghizzoni L, et al. Ketoacidosis and hyperosmolality as first symptoms of type 1 diabetes mellitus following ingestion of high-carbohydrate-containing fluids. J Pediatr Endocrinol Metab 1999;12:691-4

6 Kitabchi AE, Matteri R, Murphy MB. Optimal insulin delivery in diabetic ketoacidosis (DKA) and hyperglycemic, hyperosmolar nonketotic coma (HHNC). Diabetes Care 1982;5:78-87.

7 Luzi L, Barrett EJ, Groop LC, et al. Metabolic effects of low-dose insulin therapy on glucose metabolism in diabetic ketoacidosis. Diabetes 1988;37:1470-7.

8 Tattersall RB. A paper which changed clinical practice (slowly). Jacob Holler on potassium deficiency in diabetic acidosis (1946). Diabet Med 1999;16:987-94.

9 Duck SC, Wyatt DT. Factors associated with brain herniation in the treatment of diabetic ketoacidosis. J Pediatr 1988:113:10-14.

10 Harris GA, Fiordalisi I, Harris WL, et al. Minimizing the risk of brain herniation during treatment of diabetic ketoacidaemia: a retrospective and prospective study. J Pediatr 1990;117:22-31.

11 Harris GA, Fiordalisi I. Physiologic management of diabetic ketoacidemia. A 5 -year prospective pediatric experience in 231 episodes. Arch Pediatr Adolesc Med 1994; 148: 1046-52.

12 Edge JA, Hawkins MM, Winter DL, et al. The risk and outcome of cerebral oedema developing during diabetic ketoacidosis. Arch Dis Child 2001;85:16-22.

13 Edge JA. Cerebral oedema during treatment of diabetic ketoacidosis: are we any nearer finding a cause? Diabetes Metab Rev 2000;16:316-24.

14 Edge JA, Dunger DB. Variations in the management of diabetic ketoacidosis in children. Diabet Med 1994;11:984-6. 